\title{
SURFACES OF GLOBALLY F-REGULAR TYPE ARE OF FANO TYPE
}

\author{
SHINNOSUKE OKAWA
}

\begin{abstract}
We prove that a projective surface of globally $F$-regular type defined over a field of characteristic zero is of Fano type.
\end{abstract}

\section{INTRODUCTION}

Projective varieties of Fano type form a natural and important class of varieties from the point of view of the minimal model theory (see Definition 2.2 for definition). In this paper, we discuss a conjectural characterization of this class proposed by Schwede and Smith:

Conjecture 1.1 (= SS10, Question 7.1]). Let $X$ be a normal projective variety defined over a field of characteristic zero. Then $X$ is of Fano type if and only if it is of globally F-regular type.

For the context behind this conjecture, see [SS10]. The 'only if' direction is already proven in [SS10, Theorem 1.2], as an application of [Tak04, Corollary 3.4]. That is, a variety of Fano type defined over a field of characteristic zero is of globally $F$-regular type. Aiming at the other direction, the authors constructed a boundary divisor $\Delta$ for any globally $F$-regular variety $X$ (defined in a fixed positive characteristic) such that $(X, \Delta)$ is $\log$ Fano [SS10, Theorem 4.3 (i)]. The difficulty lies in that it is not clear if the boundary divisor $\Delta$ can be lifted to characteristic zero or not.

On the other hand, it was proven in GOST15, Theorem 1.2] that a Mori dream space of globally $F$-regular type is of Fano type. Note that since a variety of Fano type in characteristic zero is a Mori dream space by [BCHM10, Corollary 1.3.2], if Conjecture 1.1 was solved affirmatively, then it would imply that a variety of globally $F$-regular type is a Mori dream space.

The purpose of this paper is to prove Conjecture 1.1 in dimension 2, without assuming $X$ is a Mori dream space:

Date: September 25, 2021.

2010 Mathematics Subject Classification. Primary 14J45; Secondary 14E30.

Key words and phrases. Varieties of Fano type, Varieties of globally F-regular type, Mori dream space. 
Theorem 1.2. Let $X$ be a projective surface defined over a field of characteristic zero. Suppose that $X$ is of globally F-regular type. Then $X$ is of Fano type.

Here we describe the outline of our proof. By Lemma 2.3 and Lemma 2.4, it is enough to show that $X$ admits a $\left(-K_{X}\right)$-MMP whose final model is a weak Fano variety. Note that in the proof of GOST15, Theorem 1.2], the existence of such a $\left(-K_{X}\right)$-MMP was guaranteed by the assumption that $X$ is a Mori dream space.

To show the existence of such a nice MMP, we first check in Proposition 2.8 that a globally $F$-regular surface (defined in a fixed positive characteristic) is a Mori dream space. This implies that $X_{\mu}$, the reduction of our $X$ to a positive characteristic, admits a $\left(-K_{X_{\mu}}\right)$-MMP.

Next we lift the $\left(-K_{X_{\mu}}\right)$-MMP to characteristic zero to obtain a $\left(-K_{X}\right)$-MMP (strictly speaking we have to pass to a base change of $X$ in this step, but it is a minor point). Since $X_{\mu}$ is a surface, each step of the $\left(-K_{X_{\mu}}\right)$-MMP is a divisorial contraction. Hence it is enough to show that the line bundle together with the global sections which define the morphism lift to characteristic zero (see Proposition 2.10). This follows from the standard deformation theory of line bundles (Lemma 2.9) and the vanishing theorem for cohomology of nef line bundles on globally $F$-regular varieties (Lemma 2.6). The rest of the argument is the same as that of the proof of [GOST15, Theorem 1.2].

Our proof does not use any classification result on surfaces (the author would like to thank Yoshinori Gongyo for pointing it out). In the end of this paper we clarify the obstructions which show up when we try to use our approach in higher dimensions.

After the author finished writing a draft of this paper, which is a part of the the author's PhD thesis, two relevant papers appeared. Gongyo and Takagi gave another proof for the main theorem of this paper in [GT13, and they also proved that surfaces of dense globally $F$-split type are of log Calabi-Yau type. There is also the paper [HP13, in which Hwang and Park gave yet another proof for the main theorem of this paper based on results of Sakai [Sak84].

Acknowledgements. The author would like to thank Osamu Fujino for informing him of the paper [Tan14]. He would also like to thank Shunsuke Takagi for his useful comments. He is grateful to the coauthors of the paper [GOST15] for the discussion during the preparation of GOST15, which was quite helpful for him to digest the contents of the paper. During the preparation of this paper, the author was partially supported by Grant-in-Aid for JSPS fellow 22-849. 


\section{Preliminaries}

For the notions of singularities in characteristic zero and Mori dream spaces which will be used below without definition, see [KM98 and [HK00] respectively. Readers can also refer to GOST15, Sections 2.1 $-2.3]$.

Definition 2.1. A normal projective variety $X$ is called a Mori dream space provided that the following conditions hold.

(1) $X$ is $\mathbb{Q}$-factorial and $\mathbf{P i c} \mathbf{C}_{X}^{0}$ has dimension zero.

(2) The nef cone of $X$ is the affine hull of finitely many semi-ample line bundles.

(3) There is a finite collection of small $\mathbb{Q}$-factorial modifications $f_{i}: X \rightarrow-\rightarrow$ $X_{i}$ such that each $X_{i}$ satisfies (11)(2) and the movable cone of $X$ is the union of the nef cones of $X_{i}$.

The symbol $\mathbf{P i c}{ }_{X}^{0}$ in (11) stands for the identity component of the Picard scheme of $X$ over $\mathbf{k}$ (see $\left[\mathrm{FGI}^{+} 05\right.$, Chapter 9] for details). To make sense of (3), note that the Weil divisors on $X_{i}$ are identified with those on $X$ via the small birational map $f_{i}$.

Consider a normal projective variety $X$ and an effective $\mathbb{Q}$-divisor $\Delta$ on $X$ such that $K_{X}+\Delta$ is $\mathbb{Q}$-Cartier. Such a pair $(X, \Delta)$ is called a (log)pair.

Definition 2.2. A pair $(X, \Delta)$ is called a log Fano pair if its singularity is at worst $\mathrm{klt}$ and $-\left(K_{X}+\Delta\right)$ is ample. A normal projective variety $X$ is said to be of Fano type if there exists an effective $\mathbb{Q}$-divisor $\Delta$ on $X$ such that $(X, \Delta)$ is a log Fano pair. $X$ is called a weak Fano variety if it admits at worst log terminal singularities and $-K_{X}$ is nef and big.

We will repeatedly use

Lemma 2.3 (= GOST15, Remark 2.8]). A weak Fano variety is a variety of Fano type.

The following claim, whose proof is implicitly given in the proof of GOST15, Theorem 3.2], will be repeatedly used in this paper.

Lemma 2.4. Let

$$
X \rightarrow X_{1} \rightarrow-\rightarrow \cdots-\rightarrow X_{\ell}
$$

be a $\left(-K_{X}\right)-M M P$ such that $X_{\ell}$ is a variety of Fano type. Then so is $X$.

Next we explain about singularities in positive characteristics. Let $R$ be a commutative algebra defined over a field of characteristic $p>0$ such that the (absolute) Frobenius map $F: R \rightarrow R$ is finite. By an 
abuse of notation, the corresponding morphism between the spectra will be also denoted by

$$
F: \operatorname{Spec} R \rightarrow \operatorname{Spec} R \text {. }
$$

The finiteness of $F$ is preserved under morphisms of finite type, and hence it comes for free if we are dealing with algebraic schemes over a perfect field. For an $R$-module $M$, the $e$-times pushforward $F_{*}^{e} M$ is isomorphic to $M$ as an abelian group, whereas the action of $R$ is twisted as

$$
r \cdot F_{*}^{e} m=F_{*}^{e}\left(r^{p^{e}} m\right) \in F_{*}^{e} M .
$$

In the rest of paper, the element $F_{*}^{e} m \in F_{*}^{e} M$ will be simply denoted by $m$ if it can be read off from the context.

Definition 2.5. (i) An $F$-finite ring $R$ of characteristic $p>0$ is strongly $F$-regular if for every $c \neq 0 \in R$, there exists an integer $e \geq 1$ such that the homomorphism of $R$-modules

$$
c F^{e}: R \stackrel{F^{e}}{\longrightarrow} F_{*}^{e} R \stackrel{F_{*}^{e}(c \cdot)}{\longrightarrow} F_{*}^{e} R ; \quad a \mapsto c a^{p^{e}}
$$

splits (i.e. admits a left inverse) as a homomorphism of $R$-modules.

(ii) Let $X$ be a variety defined over an $F$-finite field of characteristic $p>0$. We say that $X$ is globally $F$-regular if for every effective divisor $D$ on $X$, there exists an integer $e \geq 1$ such that

$$
s_{D} F^{e}: \mathcal{O}_{X} \rightarrow F_{*}^{e}\left(\mathcal{O}_{X}(D)\right) ; \quad a \mapsto a^{p^{e}} s_{D}
$$

splits as a morphism of $\mathcal{O}_{X}$-modules, where $s_{D} \in H^{0}\left(X, \mathcal{O}_{X}(D)\right)$ is the global section of $\mathcal{O}_{X}(D)$ defined by the effective divisor $D$.

(iii) Let $X$ be a variety defined over a field of characteristic zero. $X$ is said to be of globally $F$-regular type if there exists a model $\pi: X_{A} \rightarrow \operatorname{Spec} A$ of $X$ such that $X_{\mu}=X_{A} \otimes_{A} k(\mu)$ is globally $F$-regular for any closed point $\mu \in \operatorname{Spec} A$ (see [GOST15, page 7] for the definition of models). A ring $R$ of finite type over a field of characteristic zero is of strongly $F$-regular type if $X=\operatorname{Spec} A$ is of globally $F$-regular type.

Globally $F$-regular varieties share nice properties with varieties of Fano type. In particular, we will use the following

Lemma 2.6 ( = [Smi00, Corollary 4.3]). Let $X$ be a globally F-regular projective variety. Then for any nef line bundle $L$ on $X$, the vanishing

$$
H^{i}(X, L)=0
$$

holds for any $i>0$. 
In order to illustrate how the definition of global $F$-regularity is used in practice, we include a proof.

Proof. Pick an ample line bundle $A$ on $X$. Then $B_{n}=A \otimes L^{\otimes n}$ is ample for any $n>0$. Take $q=p^{e}$ such that $F^{e}: \mathcal{O}_{X} \rightarrow F_{*}^{e} \mathcal{O}_{X}$ splits. Taking tensor product with $B_{n}$, we see that $B_{n}$ is a direct summand of $F_{*}^{e} \mathcal{O}_{X} \otimes B_{n} \cong F_{*}^{e}\left(B_{n}^{\otimes q}\right)$. Thus we obtain the sequence of inclusions $H^{i}\left(X, B_{n}\right) \subset H^{i}\left(X, B_{n}^{\otimes q}\right) \subset H^{i}\left(X, B_{n}^{\otimes q^{2}}\right) \subset \cdots$, and this should vanish eventually by the Serre's vanishing theorem.

Now assume $A$ is effective. So far we checked $H^{i}\left(X, A \otimes L^{\otimes n}\right)=0$ for all $n>0$. On the other hand, we know $s_{A} F^{e}: \mathcal{O}_{X} \rightarrow F_{*}^{e} \mathcal{O}_{X}$ splits for some $e>0$. Taking tensor product with $L$, we obtain the inclusion $H^{i}(X, L) \subset H^{i}\left(X, A \otimes L^{\otimes q}\right)$ to conclude the proof.

Remark 2.7. For the sake of completeness, here we include a remark on $\mathbb{Q}$-factoriality of the varieties we are interested in. Log terminal singularities and strongly $F$-regular singularities are rational singularities. Hence the $\mathbb{Q}$-factoriality of surfaces with those singularities comes for free by [Lip69, Proposition 17.1].

The following proposition is the first step toward the proof of Theorem 1.2 .

Proposition 2.8. Let $X$ be a projective and globally F-regular surface defined over an F-finite field. Then $X$ is a Mori dream space.

Proof. We directly check the conditions of Definition 2.1. First of all, see Remark 2.7 for the $\mathbb{Q}$-factoriality. Since $H^{1}\left(X, \mathcal{O}_{X}\right)$, the tangent space of $\mathbf{P i c}_{X}^{0}$ at the origin, is trivial by Lemma 2.6, the condition (11) is satisfied. To see (2), note that by [SS10, Theorem 4.3 (i)] there exists an effective $\mathbb{Q}$-divisor $\Delta$ on $X$ such that $(X, \Delta)$ is a log Fano pair. By the cone and contraction theorems for surface pairs in positive characteristics (see [Tan14, Theorem 3.13] and [Tan14, Theorem 3.21] respectively), we see that the effective cone (hence the nef cone) of $X$ is rational polyhedral and that every extremal ray of the nef cone is generated by a semi-ample line bundle which defines the extremal contraction of the supporting facet of the effective cone. Finally, since $X$ is a surface, any movable divisor is semi-ample by the Zariski-Fujita theorem. Therefore the condition (3) is automatically satisfied.

We next prove the liftability of morphisms from globally $F$-regular varieties.

Lemma 2.9. Let $A$ be a complete local ring with the closed point $\mu \in$ $\operatorname{Spec} A$, and $f: X_{A} \rightarrow \operatorname{Spec} A$ a flat projective morphism. Suppose 
$H^{2}\left(X_{\mu}, \mathcal{O}_{X_{\mu}}\right)=0$, where $X_{\mu}$ is the closed fiber. Then any line bundle $L_{\mu}$ on $X_{\mu}$ extends to a line bundle $L_{A}$ on $X_{A}$ so that $\left.L_{A}\right|_{X_{\mu}} \simeq L_{\mu}$.

Proof. See $\left[\mathrm{FGI}^{+} 05\right.$, Corollary 8.5.6(a)].

Proposition 2.10. Let $A$ and $f: X_{A} \rightarrow$ Spec $A$ be as in Lemma 2.9. Assume that $X_{\mu}$ is globally $F$-regular. Suppose that there exists a morphism with connected fibers $g_{\mu}: X_{\mu} \rightarrow Y_{\mu}$ to a normal projective variety $Y_{\mu}$. Then $g_{\mu}$ lifts to a morphism with connected fibers $g_{A}: X_{A} \rightarrow Y_{A}$ over $A$, where $Y_{A}$ is a normal projective scheme over $A$.

Proof. Pick a very ample line bundle $\mathcal{O}_{Y_{\mu}}(1)$ on $Y_{\mu}$, so that the complete linear system corresponding to the line bundle $L_{\mu}=g_{\mu}^{*} \mathcal{O}_{Y_{\mu}}(1)$ defines the morphism $g_{\mu}$ : to see this use the equality $H^{0}\left(X_{\mu}, L_{\mu}\right)=$ $H^{0}\left(Y_{\mu}, \mathcal{O}_{Y_{\mu}}(1)\right)$, which follows from the assumption that $g_{\mu}$ has connected fibers.

Since $X_{\mu}$ is globally $F$-regular, we see $H^{2}\left(X_{\mu}, \mathcal{O}_{X_{\mu}}\right)=0$ by Lemma 2.6. Hence by Lemma 2.9 we obtain a line bundle $L_{A}$ on $X_{A}$ which is a lift of $L_{\mu}$.

Also, since $L_{\mu}$ is nef, we see $H^{1}\left(X_{\mu}, L_{\mu}\right)=0$ again by Lemma 2.6. Then [Har77, Chapter III, Corollary 12.9] implies $H^{1}\left(X_{A}, L_{A}\right)=0$. Applying [Har77, Chapter III, Theorem 12.11 (b)] for $i=1$ and then [Har77, Chapter III, Theorem 12.11 (a)] for $i=0$, we can check that $H^{0}\left(X_{A}, L_{A}\right)$ is a free $A$ module satisfying

$$
H^{0}\left(X_{A}, L_{A}\right) \otimes_{A} k(\mu) \stackrel{\sim}{\rightarrow} H^{0}\left(X_{\mu}, L_{\mu}\right) .
$$

Since $L_{\mu}$ is globally generated and the base locus of a linear system is closed, this implies that $L_{A}$ is globally generated and that the corresponding complete linear system defines a morphism $g_{A}^{\prime}: X_{A} \rightarrow Y_{A}^{\prime}$, which restricts to the morphism $g_{\mu}$ over $\mu \in \operatorname{Spec} A$. By taking the Stein factorization $g_{A}: X_{A} \rightarrow Y_{A}$ of $g_{A}^{\prime}$, we obtain the desired lift of $g_{\mu}$

In the final step of the proof of Theorem 1.2, we use the following fact that the property of being of Fano type descends under base field extension.

Proposition 2.11. Let $X$ be a normal projective variety defined over a field $k$, and $k \subset K$ an extension of fields. If the base change $X_{K}=$ $X \otimes_{k} K$ is a Mori dream space, so is $X$.

Proof. Note first that the condition $\operatorname{dim} \mathbf{P i c}^{0}=0$ is stable under the change of base field, because of the functorial behaviour $\mathbf{P i c}_{X_{K} / K} \cong$ $\mathbf{P i c}_{X_{k} / k} \otimes_{k} K$. Hence by the characterization of Mori dream spaces 
[HK00, Proposition 2.9], it is enough to show that a Cox ring of $X$ is of finite type over $k$.

Let $\Gamma \subset \operatorname{Div} X$ be a finitely generated group of Cartier divisors on $X$ which defines a Cox ring of $X$. Then we have the canonical isomorphism

$$
R(X, \Gamma) \otimes_{k} K \stackrel{\sim}{\rightarrow} R\left(X_{K}, \Gamma_{K}\right),
$$

where $\Gamma_{K}$ is the pull-back of $\Gamma$ to $X_{K}$.

As explained in Oka11, Lemma 3.2], the multi-section ring on the RHS of (2.8) is of finite type over $K$. Using the descent of finite generation with respect to the field extension $k \subset K$, we obtain the finite generation of $R(X, \Gamma)$.

Corollary 2.12. Let $X$ be a normal projective variety defined over a field $k$ of characteristic zero, and $k \subset K$ an extension of fields. If the base change $X_{K}=X \otimes_{k} K$ is of Fano type, so is $X$.

Proof. By [BCHM10, Corollary 1.3.2] and Proposition 2.11, $X$ is a Mori dream space. Since $-K_{X_{K}}$ is big, so is $-K_{X}$. Hence we find a $\left(-K_{X}\right)$-MMP $X=X_{0} \rightarrow X_{1} \rightarrow \cdots \cdots-\rightarrow X_{\ell}$ such that $-K_{X_{\ell}}$ is semiample and big by [HK00, Proposition 1.11(1)]. Taking the base change by $k \subset K$, we get a $\left(-K_{X_{K}}\right)$-MMP. Since $X_{K}$ is of Fano type, so are $X_{i} \otimes_{k} K$ by [GOST15, Lemma 3.1] and [FG12, Theorem 5.1]. Hence $X_{\ell}$ also has at worst log terminal singularities, which means that $X_{\ell}$ is a weak Fano variety. Finally we can use Lemma 2.4 to conclude the proof.

\section{Proof of Theorem 1.2}

Proof of Theorem 1.2. Let $\pi: X_{A} \rightarrow$ Spec $A$ be a model of $X$, so that $X_{\mu}$ is globally $F$-regular for a closed point $\mu \in \operatorname{Spec} A$. By Proposition $\left[2.8, X_{\mu}\right.$ is a Mori dream space.

Since $X_{\mu}$ is a Mori dream space, there exists a $\left(-K_{X_{\mu}}\right)$-MMP

$$
X_{\mu}=X_{\mu, 0} \rightarrow X_{\mu, 1} \rightarrow \cdots \rightarrow X_{\mu, \ell}
$$

such that $-K_{X_{\mu, \ell}}$ is semi-ample and big by [HK00, Proposition 1.11(1)]. Since each morphism is a projective morphism with connected fibers between normal varieties, which in particular is an algebraic fiber space, we see that $X_{\mu, i}$ are also globally $F$-regular by [GOST15, Lemma 2.14].

Let $A_{\mu}$ be the localisation of $A$ at $\mu$. Consider its completion $\widehat{A}$ at the maximal ideal, and take the base change of $X_{A} \rightarrow \operatorname{Spec} A$ by $A \subset \widehat{A}$. In the rest, we will write $A$ to mean $\widehat{A}$.

The generic (resp. closed) point of $\operatorname{Spec} A(=\widehat{A})$ will be denoted by $\xi$ (resp. $\mu$ ). Note that the generic fiber $X_{\xi}$ is still of globally $F$-regular type. 
By Proposition 2.10, the morphisms in (3.1) lift over Spec $A$. They will be denoted by $X_{A}=X_{A, 0} \rightarrow X_{A, 1} \rightarrow \cdots \rightarrow X_{A, \ell}$. Since $-m K_{X_{\mu, \ell}}$ is globally generated for sufficiently divisible $m$, by the same arguments as in the proof of Proposition 2.10, we see that all the global sections of $-m K_{X_{\mu, \ell}}$ lift over $\operatorname{Spec} A$. Thus, restricting to the generic fiber, we obtain a $\left(-K_{X_{\xi}}\right)$-MMP $X_{\xi}=X_{\xi, 0} \rightarrow X_{\xi, 1} \rightarrow \cdots \rightarrow X_{\xi, \ell}$ such that $-K_{X_{\xi, \ell}}$ is semi-ample and big.

Using [GOST15, Lemma 2.14], we see that $X_{\xi, \ell}$ is also of globally $F$-regular type. Hence by GOST15, Proposition 2.17], it has only log terminal singularities. Thus we see that it is a weak Fano variety. Finally we can use Lemma 2.3 and Lemma 2.4 to conclude the proof.

\section{Concluding Discussion: TOWARD Higher DIMENSIONS}

When we try to generalize our result to higher dimensions, the following issues show up. First of all, the existence of an anti-canonical MMP was essential in our arguments. For that purpose we have to settle the following question affirmatively.

Question 4.1. Is a projective and globally $F$-regular variety over a finite field always a Mori dream space?

Now let $X$ be a projective variety of globally $F$-regular type. If Question 4.1 is answered affirmatively, then we obtain an anti-canonical MMP for a reduction $X_{\mu}$ of $X$ to a positive characteristic ending up with a weak log Fano model. Suppose that $f_{\mu, n}: X_{\mu, n} \rightarrow X_{\mu, n+1}$ is a step of such an anti-canonical MMP. If it is a divisorial contraction, we can lift it to characteristic zero as before.

Suppose that it is a $\left(-K_{X_{\mu, n}}\right)$-flip. In this case we can still lift the flipping contraction, say $X_{\mu, n} \rightarrow Y_{\mu}$, to characteristic zero. Let $g_{A}: X_{A, n} \rightarrow Y_{A}$ be the $\left(-K_{X_{A, n}}\right)$-flipping contraction thus obtained. Now the question is if one can lift the flip $X_{\mu, n+1} \rightarrow Y_{\mu}$ to characteristic zero as well.

The existence of the $\left(-K_{X_{\mu, n}}\right)$-flip is equivalent to the finite generation of the $\mathcal{O}_{Y_{\mu}}$-algebra

$$
\bigoplus_{m \geq 0} g_{\mu, n *} \mathcal{O}_{X_{\mu, n}}\left(-m K_{X_{\mu, n}}\right)=\bigoplus_{m \geq 0} \mathcal{O}_{Y_{\mu}}\left(-m K_{Y_{\mu}}\right)
$$

The corresponding statement for the existence of $\left(-K_{A, n}\right)$-flip holds as well.

If one could show that the restrictions of the sheaves $\omega_{Y_{A}}^{[m]}=\left(\omega_{Y_{A}}^{m}\right)^{* *}$ to $Y_{\mu}$ are reflexive for all $m$ which is divisible by a fixed integer $N>0$, 
then that would imply that the natural homomorphism

$$
\bigoplus_{m \geq 0, N \mid m} \mathcal{O}_{Y_{A}}\left(-m K_{Y_{A}}\right) \rightarrow \bigoplus_{m \geq 0, N \mid m} \mathcal{O}_{Y_{\mu}}\left(-m K_{Y_{\mu}}\right)
$$

is surjective. Then, using the Nakayama's lemma, we can derive the desired finite generation from that of (4.1).

Unfortunately the author does not see how to verify the necessary reflexivity.

\section{REFERENCES}

[BCHM10] Caucher Birkar, Paolo Cascini, Christopher D. Hacon and James McKernan, Existence of minimal models for varieties of log general type, J. Amer. Math. Soc. 23 (2010), no. 2, 405-468. MR 2601039 (2011f:14023)

[FG12] Osamu Fujino and Yoshinori Gongyo, On canonical bundle formulas and subadjunctions, Michigan Math. J. 61 (2012), no. 2, 255-264. MR 2944479

$\left[\mathrm{FGI}^{+} 05\right]$ Barbara Fantechi, Lothar Göttsche, Luc Illusie, Steven L. Kleiman, Nitin Nitsure and Angelo Vistoli, Fundamental algebraic geometry, Grothendieck's FGA explained, Mathematical Surveys and Monographs, vol. 123, American Mathematical Society, Providence, RI, 2005. MR 2222646 (2007f:14001)

[GOST15] Yoshinori Gongyo, Shinnosuke Okawa, Akiyoshi Sannai and Shunsuke Takagi, Characterization of varieties of Fano type via singularities of Cox rings, J. Algebraic Geom. 24 (2015), no. 1, 159-182. MR 3275656

[GT13] Yoshinori Gongyo and Shunsuke Takagi, Surfaces of globally F-regular and F-split type, preprint arXiv:1305.3056 (2013).

[Har77] Robin Hartshorne, Algebraic geometry, Springer-Verlag, New York, 1977, Graduate Texts in Mathematics, No. 52. MR 0463157 (57 \#3116)

[HK00] Yi Hu and Sean Keel, Mori dream spaces and GIT, Dedicated to William Fulton on the occasion of his 60th birthday, Michigan Math. J. 48 (2000), 331-348. MR 1786494 (2001i:14059)

[HP13] DongSeon Hwang and Jinhyung Park, Characterization of log del pezzo pairs via anticanonical models, Mathematische Zeitschrift (2013), 1-19.

[KM98] János Kollár and Shigefumi Mori, Birational geometry of algebraic varieties, With the collaboration of C. H. Clemens and A. Corti, Translated from the 1998 Japanese original, Cambridge Tracts in Mathematics, vol. 134, Cambridge University Press, Cambridge, 1998. MR 1658959 (2000b:14018)

[Lip69] Joseph Lipman, Rational singularities, with applications to algebraic surfaces and unique factorization, Inst. Hautes Études Sci. Publ. Math. (1969), no. 36, 195-279. MR 0276239 (43 \#1986)

[Oka11] Shinnosuke Okawa, On images of mori dream spaces, preprint arXiv:1104.1326 (2011).

[Sak84] Fumio Sakai, Anticanonical models of rational surfaces, Math. Ann. 269 (1984), no. 3, 389-410. MR 761313 (85m:14058)

[Smi00] Karen E. Smith, Globally F-regular varieties: applications to vanishing theorems for quotients of Fano varieties, Dedicated to William Fulton 
on the occasion of his 60th birthday, Michigan Math. J. 48 (2000), 553-572. MR 1786505 (2001k:13007)

[SS10] Karl Schwede and Karen E. Smith, Globally F-regular and log Fano varieties, Adv. Math. 224 (2010), no. 3, 863-894. MR 2628797 (2011e:14076)

[Tak04] Shunsuke Takagi, An interpretation of multiplier ideals via tight closure, J. Algebraic Geom. 13 (2004), no. 2, 393-415. MR 2047704 (2005c:13002)

[Tan14] Hiromu Tanaka, Minimal models and abundance for positive characteristic log surfaces, Nagoya Math. J. 216 (2014), 1-70. MR 3319838

Department of Mathematics, Graduate School of Science, Osaka University, Machikaneyama 1-1, Toyonaka, Osaka, 560-0043, Japan.

e-mail address : okawa@math.sci.osaka-u.ac.jp 